\title{
Certain Topological Qualities of Convex Sets in Euclidean Space
}

\author{
NEBOJŠA ELEZ
}

\begin{abstract}
In this paper, we give four theorems relevant to certain topological qualities of the convex sets in Euclidean space. All results remain to hold in any locally convex space. They show that pair of convex sets under definite conditions satisfies some supplement qualities.
\end{abstract}

\section{INTRODUCTION}

Let us denote by $\bar{A}$, Int $A$ and Fr $A$ the closure, interior and boundary of set $A$ in topological space. In a vector space $\overline{x y}$ represents a segment with endpoints $x$ and $y$, and $\Delta x y z$ represents a triangle with vertices $x, y$ and $z$.

We cite two following lemmas first from [2] and second from [1], because we will use them later.

Lemma 1. Let $A$ be convex set in topological vector space. If $x \in \bar{A}$ and $y \in \operatorname{Int} A$, then the interior points of $\overline{x y}$ belong to $\operatorname{Int} A$.

Lemma 2. For any two sets $A$ and $B$ in topological space, holds

$\operatorname{Fr} A \cup \operatorname{Fr} B=\operatorname{Fr}(A \cup B) \cup \operatorname{Fr}(A \cap B) \cup(\operatorname{Fr} A \cap \operatorname{Fr} B)$.

\section{MAIN RESUltS}

Theorem 1. For any two convex neighborhoods $A$ and $B$ of 0 , in Euclidean space, following conditions hold:

1) $\overline{A \cap B}=\bar{A} \cap \bar{B}$,

2) $\operatorname{Int}(A \cup B)=\operatorname{Int} A \cup \operatorname{Int} B$.

Proof. 1) Since $A$ and $B$ are neighborhoods of 0 , then $0 \in \operatorname{Int} A \cap$ Int $B=\operatorname{Int}(A \cap B)$. Let $x \in \bar{A} \cap \bar{B}$. The interior points of the segment $\overline{0 x}$ belong to $A \cap B$, because of Lemma 1 . The point $x$ belongs to closure of the open segment $\overline{0 x}$, so $x \in \overline{A \cap B}$. It means that $\bar{A} \cap \bar{B} \subseteq \overline{A \cap B}$. It is evident that $\overline{A \cap B} \subseteq \bar{A} \cap \bar{B}$.

2010 Mathematics Subject Classification. Primary: 46A03.

Key words and phrases. Convex subsets of Euclidean space. 
2) Suppose that Int $A \cup \operatorname{Int} B \neq \operatorname{Int}(A \cup B)$. Then there exists a point $x$ such that $x \in \operatorname{Int}(A \cup B)$ and $x \notin \operatorname{Int} A \cup \operatorname{Int} B$. Let $L=\{\lambda x$ : $\lambda>1\}$ and $y \in L$. Then the point $x$ belongs to the interior of the segment $\overline{0 y}$. Since $x \notin \operatorname{Int} A \cup \operatorname{Int} B$, because of Lemma 1, it holds that $y \notin \bar{A} \cup \bar{B}$. The point $x$ belongs to the closure of the set $\{\lambda x: \lambda>1\} \subseteq X \backslash(A \cup B)$. Consequently,

$$
L \subseteq X \backslash(\bar{A} \cup \bar{B}) \subseteq X \backslash(A \cup B) .
$$

But

$$
x \in \bar{L} \subseteq \overline{X \backslash(A \cup B)}=X \backslash \operatorname{Int}(A \cup B),
$$

which is contradiction.

Theorem 2. For any two convex neighborhoods $A$ and $B$ of 0 , in Euclidean space $X$, with nonempty intersection, following holds:

1) $\operatorname{Fr} A \cap \operatorname{Fr} B=\operatorname{Fr}(A \cup B) \cap \operatorname{Fr}(A \cap B)$,

2) $\operatorname{Fr} A \cup \operatorname{Fr} B=\operatorname{Fr}(A \cup B) \cup \operatorname{Fr}(A \cap B)$.

Proof. 1) Let $x$ be a point such that $x \in \operatorname{Fr} A \cap \operatorname{Fr} B$. Then $x \notin \operatorname{Int} A \cup$ Int $B$. Let $L=\{\lambda x: \lambda>1\}$. In the same way as in the Theorem 1 , we prove that $x \in \bar{L} \subseteq \overline{X \backslash(A \cup B)}$. Since $x \in \bar{A} \cap \bar{B}$, because of Lemma 1 , the open segment $\overline{0 x}$ belongs to $A \cap B$. Since the point $x$ belongs to the closure of the open segment $\overline{0 x}$, we have $x \in \overline{A \cap B}$. It means that

$$
\begin{aligned}
x & \in \overline{A \cap B} \cap \overline{X \backslash(A \cup B)} \\
& =\overline{A \cap B} \cap \overline{X \backslash(A \cap B)} \cap \overline{A \cup B} \cap \overline{X \backslash(A \cup B)} \\
& =\operatorname{Fr}(A \cap B) \cap \operatorname{Fr}(A \cup B)= \\
& =\overline{A \cap B} \cap \overline{X \backslash(A \cup B)} \subseteq \bar{A} \cap \bar{B} \cap \overline{X \backslash A} \cap \overline{X \backslash B} \\
& =\operatorname{Fr} A \cap \operatorname{Fr} B .
\end{aligned}
$$

2) Proof follows from 1) and Lemma 2.

Following example shows that condition of convexity of the sets in the preceding theorems can't be omitted.

Example 1. Let $t$ be interior point of triangle with vertices $x, y, z$. Let $A$ be union of triangles $\Delta x t y$ and $\Delta x z t$, and let $B$ be interior of the union of triangles $\Delta y t z$ and $\Delta x z t$. It's easy to see that:

$$
\overline{A \cap B} \neq \bar{A} \cap \bar{B},
$$

$\operatorname{Int}(A \cup B) \neq \operatorname{Int} A \cup \operatorname{Int} B$,

$\operatorname{Fr} A \cap \operatorname{Fr} B \neq \operatorname{Fr}(A \cup B) \cap \operatorname{Fr}(A \cap B), \quad$ and

$\operatorname{Fr} A \cup \operatorname{Fr} B \neq \operatorname{Fr}(A \cup B) \cup \operatorname{Fr}(A \cap B)$. 
Theorem 3. Let $A$ and $B$ be convex sets in Euclidean space $X$, such that $A \cap B$ is bounded set, dimension of whom is greater then 1 and $\overline{A \cap B} \subseteq$ Int $(A \cup B)$. Then $A \subseteq B$ or $B \subseteq A$.

Proof. Let us suppose the contrary, that the sets $A \backslash B$ and $B \backslash A$ are nonempty. Let $a \in A \backslash B, b \in B \backslash A$ and $c \in A \cap B$ be three points which do not belong to one line. These points exist because the set $A \cap B$ does not belong to one line. Let us regard the sets $A$ and $B$ in a subspace of plane $\pi$ defined by the points $a, b$ and $c$. In the plane $\pi$ holds $\overline{A \cap B} \subseteq \operatorname{Int}(A \cup B)$. Since $\overline{A \cap B}$ is compact set, there exists a number $r>0$, such that

$$
\mathrm{K}(A \cap B, r)=\{x: \mathrm{d}(x, A \cap B)<r\} \subseteq \operatorname{Int}(A \cup B) .
$$

Let be

$$
C=\mathrm{K}(A \cap B, r) \backslash \overline{A \cap B} .
$$

Let us prove that $A \cap C \neq \emptyset \neq B \cap C$.

If $A \cap C=\emptyset$, then $A \subseteq \overline{A \cap B}$, because $A$ is the convex set and $C$ is connected set, homeomorphic to an open cyclic ring. Let $x$ be any point of the set $A$. Then the line, which passes through the points $x$ and $c$ intersects the set $C$. Let $y$ be any point of this intersection. It holds

$$
C \subseteq \operatorname{Int}(A \cup B) \subseteq A \cup B
$$

and

$$
y \in C \subseteq B .
$$

The point $x$ belongs to the interior of $\overline{c y}$ and $x \in B$ because of Lemma 1 . It means $A \subseteq B$, which is contradiction. It holds $A \cap C \neq \emptyset \neq B \cap C$.

$C \subseteq A \cup B$ is connected set. Therefore, $C=(A \cap C) \cup(B \cap C)$.

The sets $A \cap C$ and $B \cap C$ are not clopen sets in $C$. Consequently,

$$
\emptyset \neq \overline{A \cap C} \cap \overline{B \cap C} \cap C \subseteq \bar{A} \cap \bar{B} \cap C .
$$

Let $z \in \bar{A} \cap \bar{B} \cap C$. Then, the open segment $\overline{z c}$ belongs to the set $A \cap B$ because of Lemma 1, but it intersects also the set $C$, what is the contradiction.

We'll show by example that dimension of intersection of sets in preceding theorem must be grater then 1 in order for theorem to hold.

Example 2. Let $A=(0,2)$ and $B=(1,3)$. Then $\overline{A \cap B} \subseteq(A \cup B)^{\circ}, A$ and $B$ are convex, but $A \backslash B \neq \emptyset$ and $B \backslash A \neq \emptyset$.

Theorem 4. If closed convex subsets $A$ and $B$ of Euclidean space $X$ have nonempty intersection, then $A \cup B$ is convex if $\overline{A \backslash B} \cap \overline{B \backslash A}=\emptyset$. 
Proof. Let us suppose that the set $A \cup B$ is not convex. Then, points $a, b \in$ $A \cup B$ and $c \notin A \cup B$ exist such that $c$ belongs to $\overline{a b}$. The both points $a$ and $b$ evidently are not from one of the sets $A$ and $B$, and we can suppose that $a \in A \backslash B$ and $b \in B \backslash A$.

Let $x$ be a point such that $x \in A \cap B$. The point $x$ does not belong to the line defined by the points $a$ and $b$, because in this case the point $c$ would belong to $\overline{a x}$ or $\overline{b x}$, i.e. to set $A$ or to set $B$. It means that the line $a b$ does not intersect the set $A \cap B$.

Let us regard the sets $A$ and $B$ in subspace of the triangle $\Delta a b x$. The set $A \cap B$ in the triangle $\Delta a b x$ is compact and then exists a point $y$ from $A \cap B \cap \Delta a b x$, which is the closest point to $\overline{a b}$. Interior points of the segment $\overline{a y}$ must belong to $A \backslash B$, otherwise there would exist point $z \in \overline{a y}^{\circ} \cap A \cap B$ and it would be closer to segment $\overline{a b}$ than $y$.

Analogously, interior points of the segment $\overline{b y}$ belong to $B \backslash A$.

From here it follows that $y \in \overline{A \backslash B} \cap \overline{B \backslash A}$, a contradiction.

Following example shows that condition $\overline{A \backslash B} \cap \overline{B \backslash A}=\emptyset$, in preceding theorem, is not necessary.

Example 3. Let $a, b, c$ and $d$ be points on the line $p$, s.t. $a-b-c-d$, and let $x$ and $y$ be points not belonging to line $p$, s.t. $\overline{x y}$ and $\overline{b c}$ are diagonals of convex quadrilateral bxcy. Let $A$ be convex quadrilateral $a x c y$, and $B$ convex quadrilateral $b x d y$. Then, $A \cup B$ is convex quadrilateral axdy, but $\overline{A \backslash B} \cap \overline{B \backslash A}=\{x, y\} \neq \emptyset$.

Remark 1. From the proofs of the theorems we can conclude that all theorems remain to hold in any locally convex space.

\title{
REFERENCES
}

[1] K. Kuratowski, Topology, Vol. 1, Academic Press, 1966.

[2] Schaefer, Helmuth H., Topological vector spaces, New York: Springer-Verlag, 1971.

\author{
Nebojs̃a Elez \\ Faculty of Philosophy \\ AleKse ŠAntića 1 \\ 71420 PALE \\ Bosnia And Herzegovina \\ E-mail address: nelez@ffuis.edu.ba
}

